\title{
Sechs-Minuten-Gehtest Belastbarkeit bestimmt den Schweregrad der PAH
}

Ist die Diagnose pulmonale arterielle Hypertonie (PAH) gesichert, lassen die Patienten sich mithilfe des SechsMinuten-Gehtests einem WHO-Stadium zuordnen.

WHO-Stadium I - asymptomatische PAH: Die Patienten sind in ihrer körperlichen Aktivität nicht eingeschränkt. WHO-Stadium II - leichte PAH: Bei ver- stärkter körperlicher Aktivität kommen Dyspnoe, Abgeschlagenheit oder Synkopen vor.

WHO-Stadium III - mittelschwere PAH: Einschränkung schon bei leichter körperlicher Aktivität, keine Ruhedyspnoe.

WHO-Stadium IV - schwere PAH: Jede Form der Aktivität ist eingeschränkt, in Ruhe Dyspnoe und Abgeschlagenheit. Risiko für Rechtsherzversagen.

(R̈̈) $\rightarrow$ len Abklärung der Verdachtsdiagnose $\mathrm{PH}$ hat die transthorakale Echokardiografie. Die Abschätzung des pulmonalen arteriellen Drucks basiert auf der Doppler-gestützten Messung der Regurgitationsgeschwindigkeit $\left(\mathrm{V}_{\max }\right)$ über der Trikuspidalklappe. Mit der vereinfachten Bernoulli-Gleichung (DeltaP $=4 \mathrm{mal}$ $V_{\max }^{2}$ ) wird der Druckgradient zwischen dem rechten Ventrikel und dem rechten Vorhof abgeschätzt. Der rechtsatriale Druck wird üblicherweise über die Weite und Atemvariabilität der unteren Hohlvene abgeschätzt. Andere echokardiografische Kriterien sollten zur Klärung der Diagnose PH herangezogen werden. Dazu gehören ein beschleunigter Pulmonalklappenregurgitationsjet, rechtsventrikuläre Hypertrophie, verkürzte RV-Akzelerationszeit, vergrößerte rechte Herzhöhlen sowie erweiterte Pulmonalarterien.

\section{Größe und Funktion des linken Ventrikels wichtig}

Außerdem liefert die Echokardiografie wichtige Hinweise für Ursachen der $\mathrm{PH}$. Wichtig sind hier Größe und Funktion des linken Ventrikels sowie die Größe des linken Vorhofs, die auf linksventrikuläre Erkrankung oder ein Klappenvitium hinweisen können. Beim diagnostischen Vorgehen bei einer PH zielt die Strategie darauf, zunächst die häufigeren
Formen zu identifizieren (Klasse 3 Lungenerkrankungen; Klasse 2 Herzerkrankungen) und fokussiert dann auf der Unterscheidung zwischen CTEPH und den Formen der PAH.

\section{Abrechnungs-Tipp EKG-Leistungen extra vergütet}

Hausärzte rechnen bei Patienten

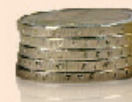

mit pulmonaler arterieller Hypertonie grundsätzlich die Versichertenpauschale und den Morbiditätszuschlag ab. Zu denken ist auch an das Belastungs-EKG (Nr. 03321) oder das Langzeit-EKG (Nrn. 03322, 03241).

Für beide Leistungen haben Hausärzte seit dem 1. Juli ein QZV zur Verfügung, sie werden also zusätzlich zum Regelleistungsvolumen vergütet. Bei Fachinternisten/Kardiologen sind diese Leistungen Bestandteil der Zusatzpauschale nach Nr. 13250 oder der Zusatzpauschale Kardiologie I nach Nr. 13545.

Gerade im hausärztlichen Bereich sollten Sie beachten, dass Ihnen ein entsprechendes QZV nur dann zur Verfügung steht, wenn Sie im Bezugsquartal mindestens eine der entsprechenden Leistungen erbracht haben.

\section{1

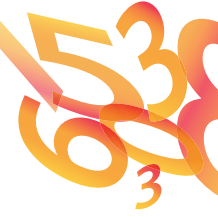 \\ Daten und Zahlen}

Das Erkrankungsalter bei pulmonaler arterieller Hypertonie liegt im Mittel bei 36 Jahren.

Das Krankheitsbild der pulmonalen Hypertonie wurde erstmalig 1868 beschrieben.

Bei drei bis fünf Patienten pro eine Million Einwohner findet sich keine Ursache für die pulmonale arterielle Hypertonie.

Nach Daten eines Registers für PAHPatienten haben etwa 32\% von ihnen eine idiopathische PAH.

Die pulmonale arterielle Hypertonie wird bei mehr als $80 \%$ erst dann diagnostiziert, wenn die Patienten im Stadium III oder IV gemäß den Kriterien der WHO sind.

Langzeitnachbeobachtungen zweier Studien mit Ambrisentan bei Patienten mit pulmonaler arterieller Hypertonie haben belegt, dass der Wirkstoff über zwei Jahre effektiv ist und gut vertragen wird (J Am Coll Cardiol 2009, 54:1971).

\section{CME Punkte}

\section{Noch mehr Lust auf Fortbildung?}

Zu dieser Sommer-Akademie-Folge stellt CME.springer.de aus dem reichhaltigen Weiterbildungsangebot ein CME-Modul bis zum 20.09.2010 kostenfrei online zur Verfügung.

Das CME-Modul „Diagnose? Leicht gemacht! Mit Anamnese und Untersuchung zum Erfolg" finden Sie auf CME.springer.de unter der Zeitschrift "CME" oder auch unter den kostenfreien Fortbildungseinheiten.

Viel Erfolg beim Punkte sammeln! 